ALICE INT-99-07

Internal Note

28 January 1999

\title{
IRRADIATION OF A SILICON-STRIP DETECTOR AND READOUT CHIPS FOR THE ALICE EXPERIMENT AT LHC
}

M. Germain, G. Guillaume, C. Suire, A. Tarchini, J.P. Coffin, L. Arnold,

J. Baudot, D. Bonnet, F. Jundt, C. Kuhn, J.R. Lutz, S. Higueret, A. Michalon

Institut de Recherches Subatomiques-IReS - IN2P3/CNRS - ULP

BP28, F 67037 Strasbourg Cedex 02 - France

W. Dulinski

Laboratoire d'Electronique et de Physique des Systèmes Instrumentaux LEPSI INQP3/CNRS - ULP BP28, F 67037 Strasbourg Cedex 02 - France

F.F. Kuntz

Aérial

19, rue de St Junien, F-67305 Schiltigheim - France

\begin{abstract}
The ALICE detector at LHC is a complex apparatus which makes use, in the internal-tracking-system part, of silicon-strip detectors. These sensors will be exposed to particle irradiation which might harm their performances as well as those of their readout electronics. In the framework of R\&D of these detectors and of their associated electronics, a module, associating a strip detector and ALICE $128 \mathrm{C}$ readout chips, has been irradiated with $20 \mathrm{MeV}$ protons beyond the dose expected to be deposited over the whole ALICE experiment $(\sim 10 \mathrm{krad}$ within about 10 years of running). Results relative to the static and dynamic characteristics of this module are presented and discussed.
\end{abstract}




\section{Introduction :}

The heavy-ion detector ALICE [1] (A Large Ion Collider Experiment) is in preparation at $\mathrm{LHC}$. It is a complex apparatus aimed at the detection of hadrons and leptons emitted in the $\mathrm{Pb}+\mathrm{Pb}$ collisions at ultra-relativistic energies. The inner component of the detector, covering the mid rapidity region $(|\eta| \leq 0.9)$, the so-called Inner Tracking System (ITS), is devoted to the tracking of the charged particles from near the interaction point up to about $45 \mathrm{~cm}$ in the transverse direction. As many of them will decay further, it allows secondary vertex reconstruction. It also permits the detection of low transverse-momentum particles, including those which do not reach the outer parts within the acceptance of the detector.

The ITS consists, from inside to outside, of six concentrical layers (centered on the beam axis) of silicon detectors of the pixel, drift and strip type, respectively, two successive layers being of the same nature. The outmost two layers are cylinders of 40 and $45 \mathrm{~cm}$ radius, for a length of 45.1 and $50.4 \mathrm{~cm}$. They associate 770 and 936 detection modules, respectively. Each cell is a double-sided silicon-strip detector (SSD). It is $7.5 \mathrm{~cm}$ long and $4.2 \mathrm{~cm}$ wide and $300 \mu \mathrm{m}$ of thickness, for a total of 768 strips, with a pitch of $95 \mu \mathrm{m}$, on each side. The strips have a stereoscopic angle of 35 milliradians, they are oriented with an angle of \pm 17 milliradians with respect to the short side of the SSD, depending on the detector side. The strips are biased at $<60$ volts by means of a polarisation ring, surrounding them, via " punch thru "technique. The whole ensemble is protected with a guard ring. The strips are readout by chips of 128 analog channels, the ALICE $128 \mathrm{C}$ chip, $([2,3])$ jointly developed by the LEPSI and the IReS de Strasbourg. Thus, each detector side is bonded to 6 chips mounted on an hybrid card.

The ALICE experiment is expected to be run over about one month per year during ten years starting in 2005. Simulations [1], currently in progress, indicates that the total irradiation of SSD modules should not exceed a total of about $10 \mathrm{krad}$. This figure resides in the domain where SSD and, more likely, chips, not radiation hard designed, might suffer damages from energy deposition from the particles going through, hence modify their performances.

In the framework of the R\&D on SSD's, we have irradiated a test structure of a SSD, designed to the ALICE specifications, but with only 128 strips on each side, bonded to two ALICE $128 \mathrm{C}$ chips, with $20 \mathrm{MeV}$ protons delivered by the VIVITRON Electrostatic-Tandem accelerator of the IReS laboratory at Strasbourg. The SSD structure has been exposed successively to 5 and $10 \mathrm{krad}$ and the chip

to a dose equivalent to $50 \mathrm{krad}$ for the SSD. Static and dynamic characteristics of this ensemble have been monitored in real time and recorded on-line. They will be presented and discussed in this article. 


\section{Experimental Setup :}

The SSD module has been installed on a spectrometer, placed in the air, intersecting the proton beam exiting from vacuum through a Kapton foil. The global scheme of the setup is illustrated in Fig. 1.

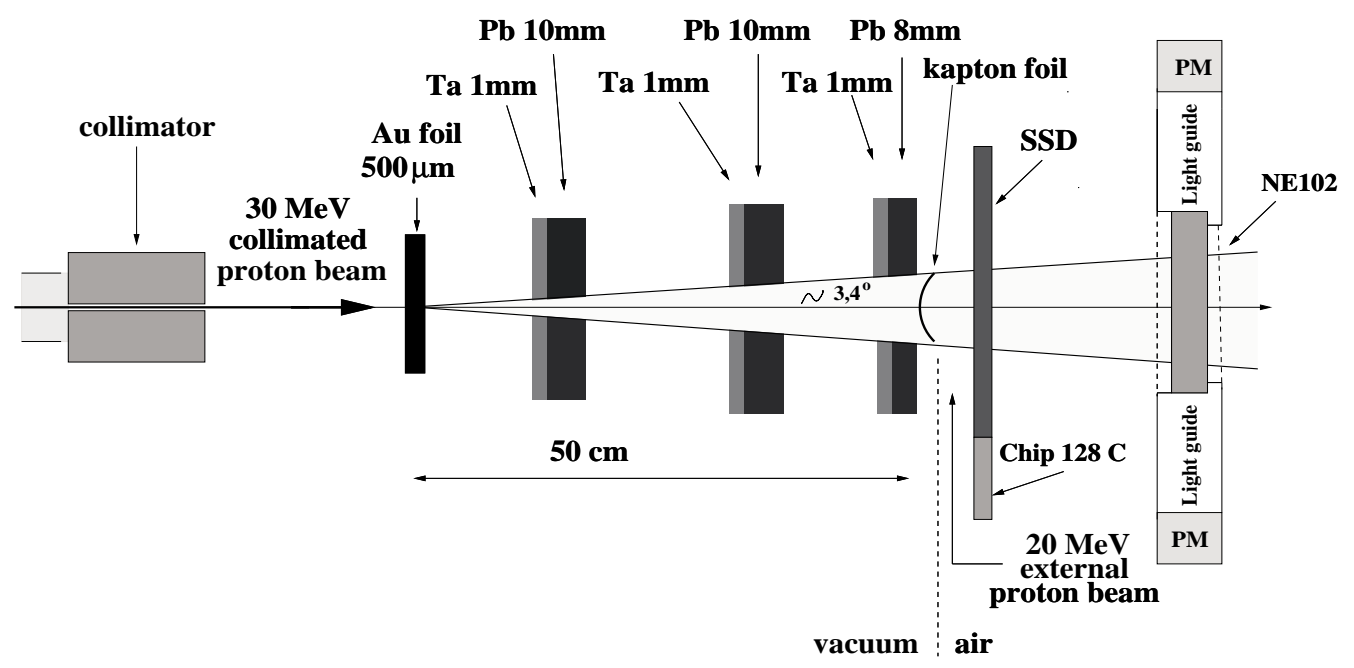

Figure 1: Scheme of the experimental setup.

\subsection{Beam Production}

A parallel proton beam of $30 \mathrm{MeV}$ and low intensity section density $\left(\sim 0.5 \mathrm{nA} / \mathrm{cm}^{2}\right)$ has been produced with the VIVITRON accelerator. This beam goes through a complex collimator reducing the beam size to a cross-section of about $3 \mathrm{~mm}^{2}$, it collides further with a thick gold foil $(500 \mu \mathrm{m})$ wich creates a strong multiple scattering [4], generating protons of roughly uniform energy $(20.3 \pm 0.2 \mathrm{MeV})$ within a cone of $\pm 30^{\circ}$. These protons traverse a second collimator, reducing the cone opening to $\pm 1.7^{\circ}$, before going through a thin Kapton foil $(50 \mu \mathrm{m})$ into the atmosphere. Considering the proton energy-loss in the foil $(0.26 \mathrm{MeV})$, and neglecting, at first order, the energy-loss over a few centimetres of air, one may irradiate objects with an external proton beam of $20 \mathrm{MeV}$ with a uniform surface density by placing them downstream the Kapton foil.

\subsection{Irradiated Module Assembly}

To prevent it from the risk of a damage a ALICE double-sided SSD was not irradiated. A so-called test structure, which is a replica of it, except that it counts 128 strips instead of 768 , was exposed. This structure was bonded to two ALICE $128 \mathrm{C}$ chips mounted on a hybrid board. The assembly is completely equivalent to that used in other circumstances [3] for testing modules, using a full size SSD connected to 12 chips, in SPS beam at CERN. A picture of the system may be found 
in Ref. 3. The assembly was placed $2 \mathrm{~cm}$ behind the Kapton foil (so allowing the operation in same bias conditions as for the ALICE experiment) and was translatable in the plan orthogonal to the beam axis in order to irradiate two different areas of the SSD test-structure and the chip separately in a third step.

\subsection{Monitoring and Trigger}

The rate of protons going through the irradiated object is measured with the help of a scintillator (NE102) of $1 \mathrm{~mm}$ thickness. It is inserted into a circular hole opened up in a cylinder of light-guide, orthogonally to its axis, which is readout at each extremity, by one photomultiplier (PM). This system is placed downstream the exposed element. The PM pulses, summed up in an AND module, are used to define the irradiating proton rate and also to trigger the data acquisition system, as described in a next section. The rate was typically of $3.5 * 10^{5}$ proton $/ \mathrm{s}$ and remained constant within about $10 \%$ during the whole irradiation. The beamimpact size on the exposed object was determined by means of a dosimetric film GAFchromic (DM-1260) placed just in front of the object in the beam path. It is sensitive to charged particles, $\mathrm{X}$ and $\gamma$-rays, and shows a bluish spot materialising the impact. By spectro photometric analyses, the cross-section of the beam was determined to be homogenous, circular, with $8 \mathrm{~mm}$ of diameter, and its centre's position located with an accuracy better than a millimeter.

The proton rate was converted into absorbed irradiation-dose by using the simple relationship :

$$
D=E \times N_{p} / m
$$

where $D$ is the absorbed dose, $E$ the energy lost by one proton in the traversed material, $N_{p}$ the number of particles counted with the scintillator during the irradiation, and $m$ the mass of the material. As a counter check, several pills of PolymerALANINE dosimeter ( $4 \mathrm{~mm}$ in diameter and $4 \mathrm{~mm}$ thick) were placed in the back of the irradiated object. They are probes which allow, via the ESR (Electronic Spin Resonance) method, the determination of the absorbed doses by the counting of the free radicals created by the ionizing particles going through [5]. The number of the created free radicals is proportionnal to the absorbed dose. The conversion factor was previously established in the domain of absorbed doses ranging from 10 up to 60 $\mathrm{krad}[6]$ and allows the determination of the absorbed dose with about $5 \%$ accuracy. A good agreement (> 90\%) was obtained between the two approaches. In view of the present goal, it is wise to notice that an evaluation of the absorbed dose with about $10 \%$ error is acceptable.

Turning now to the irradiation step concerning the read-out chip, the setup described above was modified. Indeed, the chip is implanted on the surface of a silicon bulk of $500 \mu \mathrm{m}$ thickness which is bonded on a hybrid card ( $2 \mathrm{~mm}$ thick). This yields a strong proton multiple-scattering and a substantial energy loss. As a result, the scintillator + photomultiplier ensemble was placed upstream the irradiated 
chip. For comparison of the results, all the absorbed doses were evaluated from the relationship (1) in dose equivalent to that received by the SSD in the geometry of Fig. 1.

\subsection{Data Acquisition System}

The data acquisition system is twofold and it is schematised in Fig. 2. On the one hand, different characteristics of the SSD and of the chip were recorded on-line and stored with a system controlled by LabView. The control parameters of the chip were adjusted via JTAG protocol. These characteristics were $i_{B S}$, the current measured on the polarisation structure of the SSD, $\mathrm{i}_{G}$ measured on the guard ring, $\mathrm{i}_{B+}, \mathrm{i}_{B-}$, the currents drained by the $+2 \mathrm{~V}$ and $-2 \mathrm{~V}$ power supplies of the ALICE chip, and $\mathrm{Np}$ the number of protons impinging the scintillator. These quantities were measured with $\mu \mathrm{A}$-meters and a scaler, and were read-out and stored on a PC hard-disk via the GPIB link. The irradiation proceeded during 300 seconds, then the beam was cut-off over 3 seconds during which the meters and the scaler were read-out.

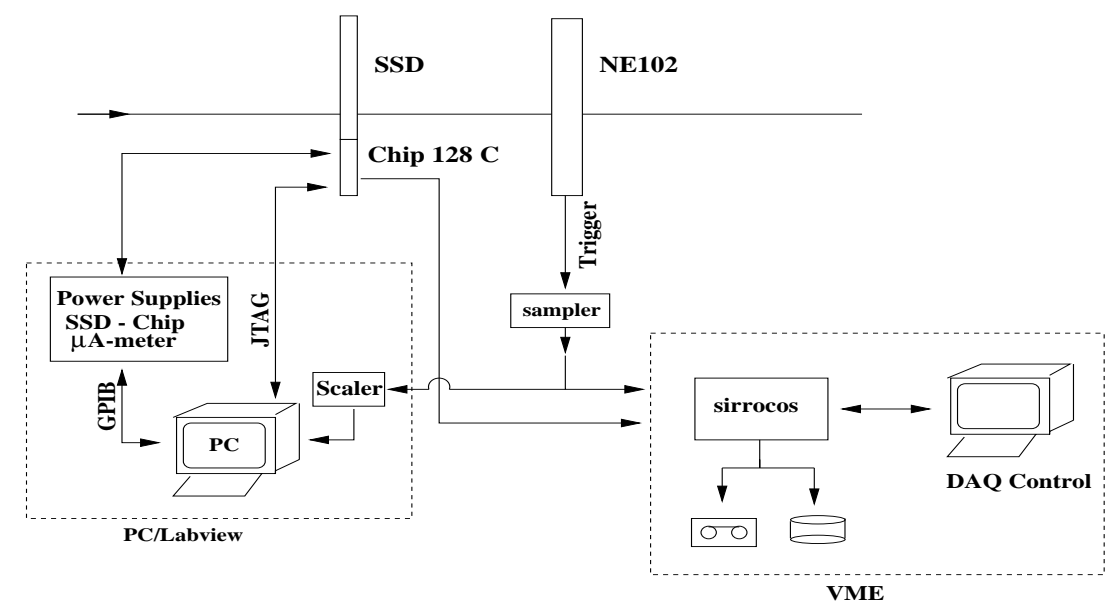

Figure 2: Scheme of the data acquisition.

On the other hand, the ALICE $128 \mathrm{C}$ read-out chips were connected to a Sirocco analog-digital converter [7]. Data control and acquisition are performed by VME electronic modules. The on-line program is based on MicroDAS software, using OS9 operating system, implemented by the LEPSI at Strasbourg. The operating condition are described in Ref. 6 . With this system, after triggering by $2^{9}$ sampled signals from the scintillator, the 128 strip analog-signals of the SSD on both sides were stored. Furthermore, pulses delivered by the internal generator [3] of the chip were fired into six different channels on each side of the SSD. In this way, it is possible to analyse the static and dynamic characteristics of the chip during the course of the irradiation and to quantify the possible changes of these characteristics. 


\section{Measurements}

Three different irradiation runs have been performed. The first two were devoted to the SSD structure and the third to the chip reading-out the p-side of the structure. In the first run, the central part of the p-side of the detector structure was exposed. In the second, it was one corner on the same side of the structure, thus allowing to examine its behavior when the polarisation structure is bombarded, and complementarily to observe a possible difference between the middle strips and those located toward an extremity of the SSD. Many strips were, however, bombarded both during the two successive exposures but in different places along their length. In view of the beam spot the whole chip area $(8 \times 6 \mathrm{~mm})$ was submitted to irradiation during the third run. The effective part of the chip was evidently oriented upstream. These runs are called IR 1,2, 3 and correspond to doses of 5, 10 and $50 \mathrm{krad}$ absorbed in $300 \mu \mathrm{m}$ of silicon, respectively.

\section{Results and discussion}

Some results about the characteristics of the charge clusters created by charged particles are presented in the next sections. They have been obtained with a computer program initially developped by the RD42 collaboration [8]. The clusters are defined as follows: First, the pedestals are calculated for a certain number of events for each strip by averaging the amplitude of raw-data signals. The residual fluctuations, after subtracting the pedestal, represents the noise characteristic of each strip. In order to determine the strips activated by the crossing of a charged particle, i.e. the charge cluster, one first looks for the strips whose the signal-to-noise ratio $(\mathrm{S} / \mathrm{N})$ exceeds a limit chosen by the user. Once, such a strip has been isolated, the neighbouring strips are examined and also retained if their own $\mathrm{S} / \mathrm{N}$ is larger than a lower threshold. This yields a strip cluster where $\mathrm{S}$ is the sum of strip signals and $\mathrm{N}$ is the arithmetical mean of the strip noises. The details of this cluster finding will be presented in a further publication dedicated to SSD beam tests [9].

Results specific to the SSD-structure, read out by the chips, will be presented first. They will be followed by those specific to the chip. Some distributions are expressed in number of electrons. They have been calibrated with the help of the chip internal generator pulses, corresponding to $11.5 \mathrm{MiP}$ 's [10, 11] where one MiP corresponds to 25000 electrons in $300 \mu \mathrm{m}$ of silicon.

\subsection{SSD-structure Results}

\subsubsection{Beam Profiles}

In order to check the correct functioning of the setup, the beam profiles were measured for IR1 and IR2. They are shown in Fig. 3 which represents the number of clusters on the 128 strips of the p-side of the SSD. Indeed in IR1, where the central 
part of the structure was irradiated, the beam profile extends roughly from channel 10 up to 100, while in the case of IR2 it is clearly shifted down by about 20 strips. The width of the IR1 profil is in agreement with the beam spot size $(0.095 \times 90=$ $8.5 \mathrm{~mm})$.
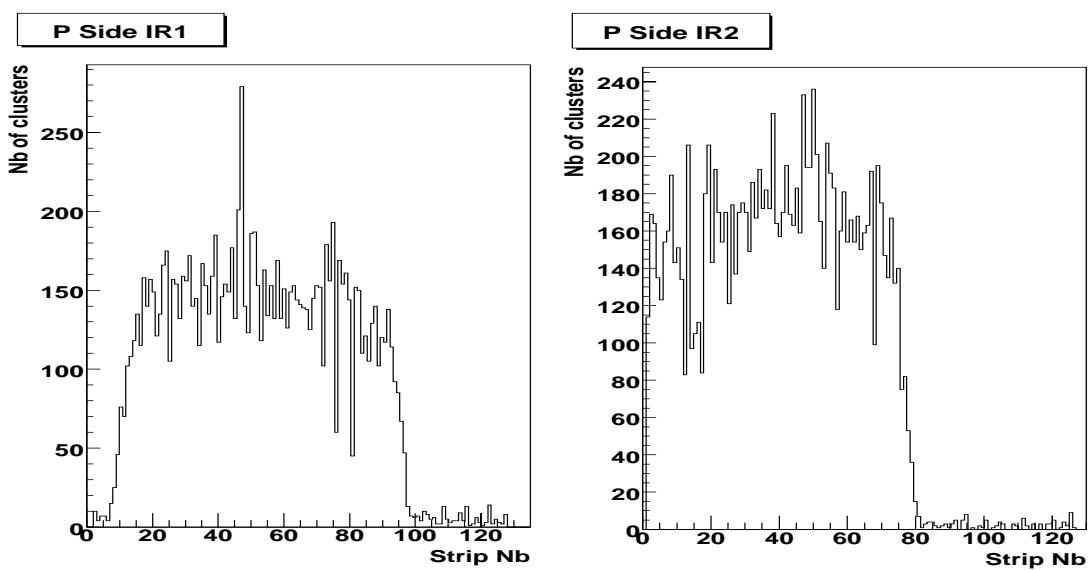

Figure 3: Beam profiles on the SSD p-side for the two irradiation runs IR1 (left) and IR2 (right).

\subsubsection{Leakage Currents}

The global leakage currents $i_{B S}$ and $i_{G}$ were measured on the bias ring and on the guard ring. The $i_{B S}$ current is plotted versus the absorbed dose in Fig. 4 for IR1 and IR2. One observes a regular linear increase for IR1 from the initial value of 140 $\mathrm{nA}$ up to about $180 \mathrm{nA}$, for a slope of $8 \mathrm{nA} / \mathrm{krad}$. During IR2, $\mathrm{i}_{B S}$ keeps increasing up to $230 \mathrm{nA}$ with a somewhat lower slope $(\sim 5 \mathrm{nA} / \mathrm{krad})$. The lowering, occuring after $10 \mathrm{krad}$, may be reliably correlated to a relatively long beam interruption which indicates that the leakage current decreases as soon as the irradiation stops. Indeed, the current decreases when the irradiation stops, it was measured equal to $200 \mathrm{nA}$ after 50 hours and to $188 \mathrm{nA}$ after 62 days (with no biasing on the detector during this period). Conversely, the sudden increase appearing at $13 \mathrm{krad}$ is due to a moderate but abrupt beam-intensity increase. 


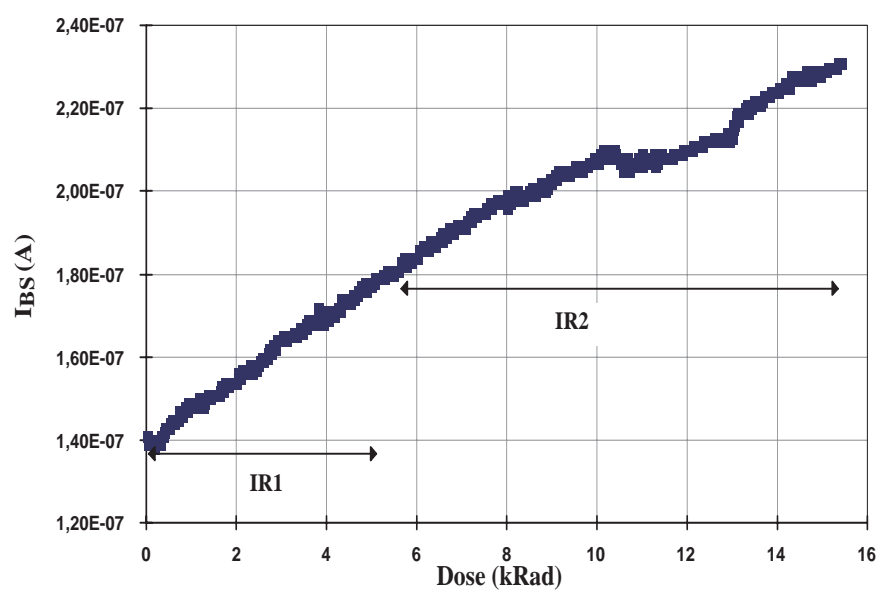

Figure 4: Leakage current measured on the bias ring $\mathrm{i}_{B S}$ of the SSD as a function of the absorbed dose.

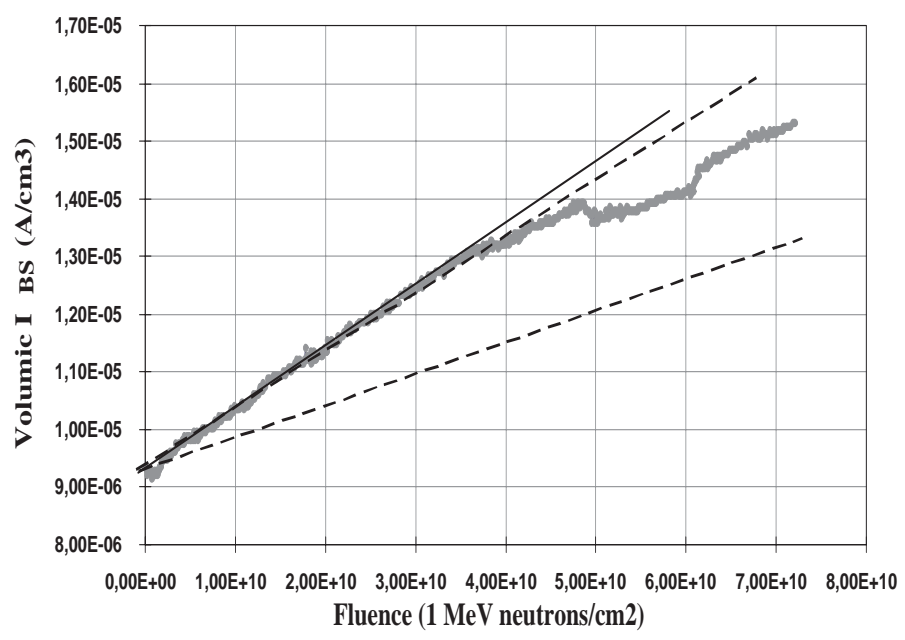

Figure 5: Volumic leakage current measured on the bias ring $i_{\text {vol }}$ as a function of the equivalent $1 \mathrm{MeV}$ neutron fluence. The solid line corresponds to a fit to the present results with a slope of $10.4^{*} 10^{-17} \mathrm{~A} / \mathrm{cm}$. The dashed lines corresponds to a linear increase of $i_{\text {vol }}$ with a slope of 5 and $10^{*} 10^{-17} \mathrm{~A} / \mathrm{cm}$ as previously observed by other authors.

Similar measurements have been performed in the past [12]-[16]. They are frequently presented in term of volumic current versus the fluence of $1 \mathrm{MeV}$ neutrons. For comparison purpose we have converted the present data to this referential, they are shown in Fig. 5. The leakage current has been calculated from $i_{B S}$ for the irradiated bulk of the SSD structure and the damages produced by $20 \mathrm{MeV}$ protons 
have been transformed in their equivalent for $1 \mathrm{MeV}$ neutrons in Silicon from Ref. [15]. It is frequently considered $[12,14]$ that the volumic current $i_{\text {vol }}$ increases as a function of the neutron fluence according to :

$$
i_{\text {vol }}\left(A / \mathrm{cm}^{3}\right)=a \cdot 10^{-17} * \text { Fluence }+b
$$

where $a$ is generally estimated to range between 5 and 10 . These two limits have been reported in Fig. 5 (dashed lines). The upper one agrees well with the current presently measured.

The leackage current $\mathrm{i}_{G}$, measured on the guard ring, is shown in Fig. 6 as a function of the absorbed dose. It also increases roughly monotonically from $2.9 \mu \mathrm{A}$ up to $3.8 \mu \mathrm{A}$, and levels off above $13 \mathrm{krad}$. The observed irregularities are correlated to fluctuations in beam intensity, particularly after long beam interruptions.

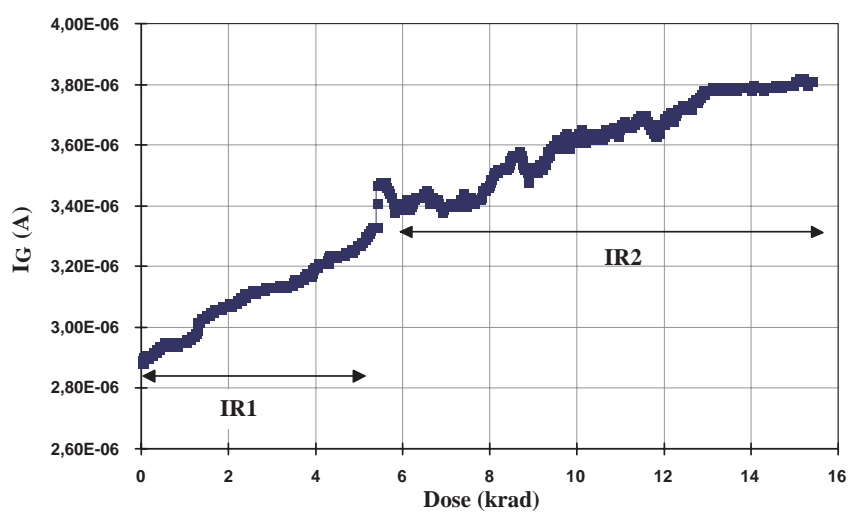

Figure 6: Leakage current $\mathrm{i}_{G}$ measured on the guard ring of the SSD as a function of the absorbed doses IR1 and IR2.

\subsubsection{Signal to Noise Ratio for the SSD-Chip module}

The signal to noise ratio $(\mathrm{S} / \mathrm{N})$ of the collected charge cluster is a relevant characteristic of the good functioning of the SSD-structure + ALICE 128C chip ensemble. This signal is generated on top of a noise specific to each strip and the corresponding read-out channel. The $\mathrm{S} / \mathrm{N}$ ratios are shown in Fig. 7 for the p-side of the SSD structure before irradiation and after IR $1+\operatorname{IR} 2(5+10 \mathrm{krad})$. Since the chip was not exposed during IR1 + IR2, the data shown here reflect the behaviour specific to the SSD structure. After irradiation, a decrease of the mean-value of $9 \%$ is observed with no other marked changes, indicating that data are not appreciably distorted by such an irradiation. Similar observation stands for the n-side. It is good to keep in mind that, in the present case, the clusters are for a high rate of particles of about $12 \mathrm{MiP}$ 's, i.e. conditions departing substantially from those which are frequently published [17] about SSD tests at much lower rate of particles 
of typically 1 MiP. As a consequence, we have to impose here higher S/N thresholds in cluster finding algorithm. This explains the sharp cut on the lower part of the distributions in Fig. 7.
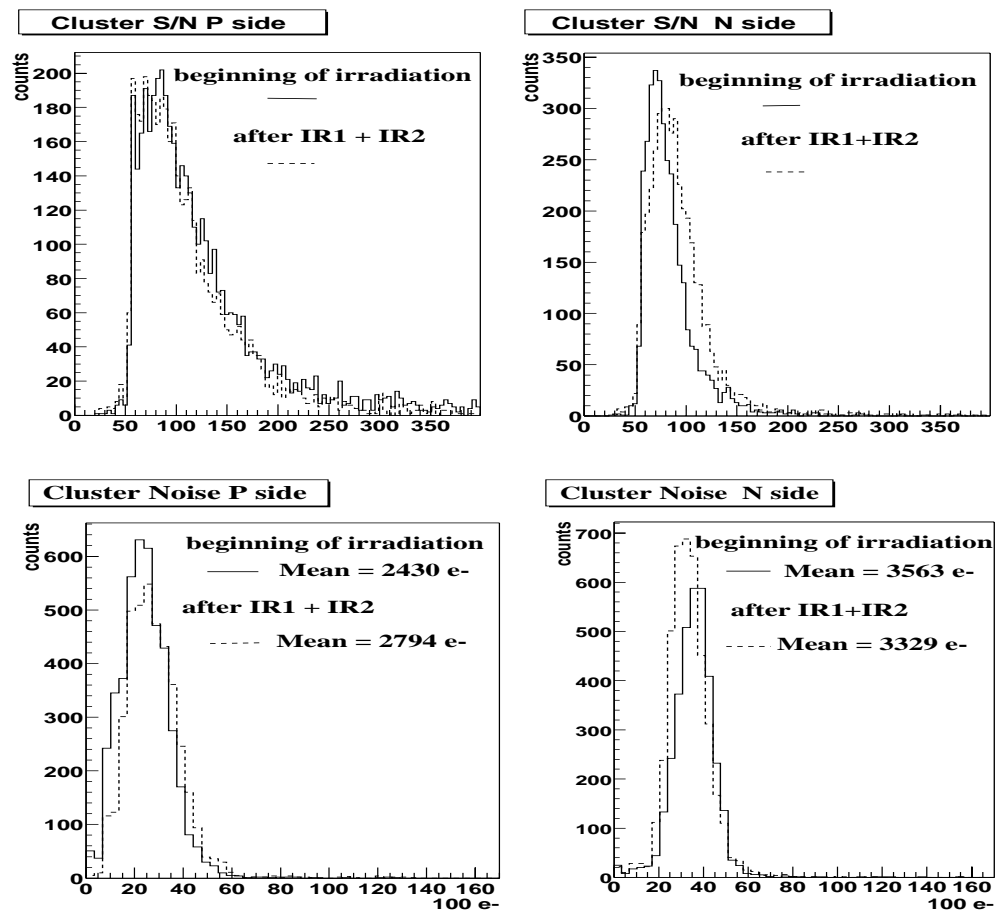

Figure 7: Clusters characteristics for $12 \mathrm{MiP}$ particles: Signal to Noise ratios (up) for p-side (left) and n-side (right). Cluster noise (down) for p-side (left) and n-side (right).

The noise distributions measured before and after irradiation (IR1+IR2) are also presented in Fig. 7, an increase of the mean value of about $14 \%$ is observed.

\subsubsection{Charge Matching in the SSD}

One particular interesting feature of the double-sided SSD is that it allows the matching of the signals collected on both sides, thus reducing the ambiguities in the case of multi-hits. Hence, it is of strong interest to examine this characteristic during IR1+IR2. A convenient observable to examine it is the ratio of the cluster charge on the $\mathrm{n}$-side $(\mathrm{Qn})$ over the cluster charge on the $\mathrm{p}$-side $(\mathrm{Qp})$ of the detector. The width of this ratio distribution is then correlated to the resolution of charge matching, since in an ideal case the charge of the cluster collected on the n-side should be proportional to that collected on the p-side. The distributions of those ratios at the beginning of IR 1 and the end of IR2 are reported in Fig. 8. These distributions are not significantly affected by irradiation. The RMS of the Qn/Qp ratio remains almost constant and the sigma of the distribution is about $15 \%$. 


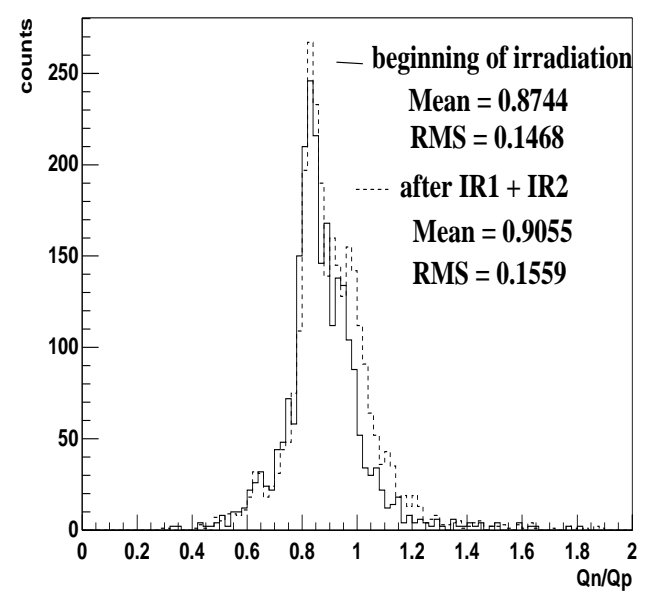

Figure 8: Qn/Qp distributions at the beginning (solid line) and the end (dashed line) of the IR $1+$ IR2 irradiation.

\subsection{ALICE 128C Chip Results}

\subsubsection{Pedestals}

Each channel of the chip is characterised by a specific pedestal whose the amplitude is typically of the order of $250 \mathrm{mV}$. The trend of pedestal level during IR1+IR2+IR3 is shown in Fig. 9. Data for four channels, randomly selected, on the chip readingout the p-side (the irradiated chip) are displayed, together, for comparison, with those for two channels on the n-side, i.e. the other chip which has not been irradiated. All remain constant, within statistical fluctuations, during IR1+IR2, as expected. In the course of IR3 the pedestals of the irradiated chip decrease subtantially from $+250 \mathrm{mV}$ down to $-80 \mathrm{mV}$, while those of the other chip remain unchanged. It is important to observe that the decrease occurs significantly after IR3 $\sim 15 \mathrm{krad}$ (beyond the lifetime of the ALICE experiment). However, after IR3 was completed, by tuning the control parameters [3] of the chip, it was possible to restore the pedestals to their nominal values, hence to the original performances of the chip. 

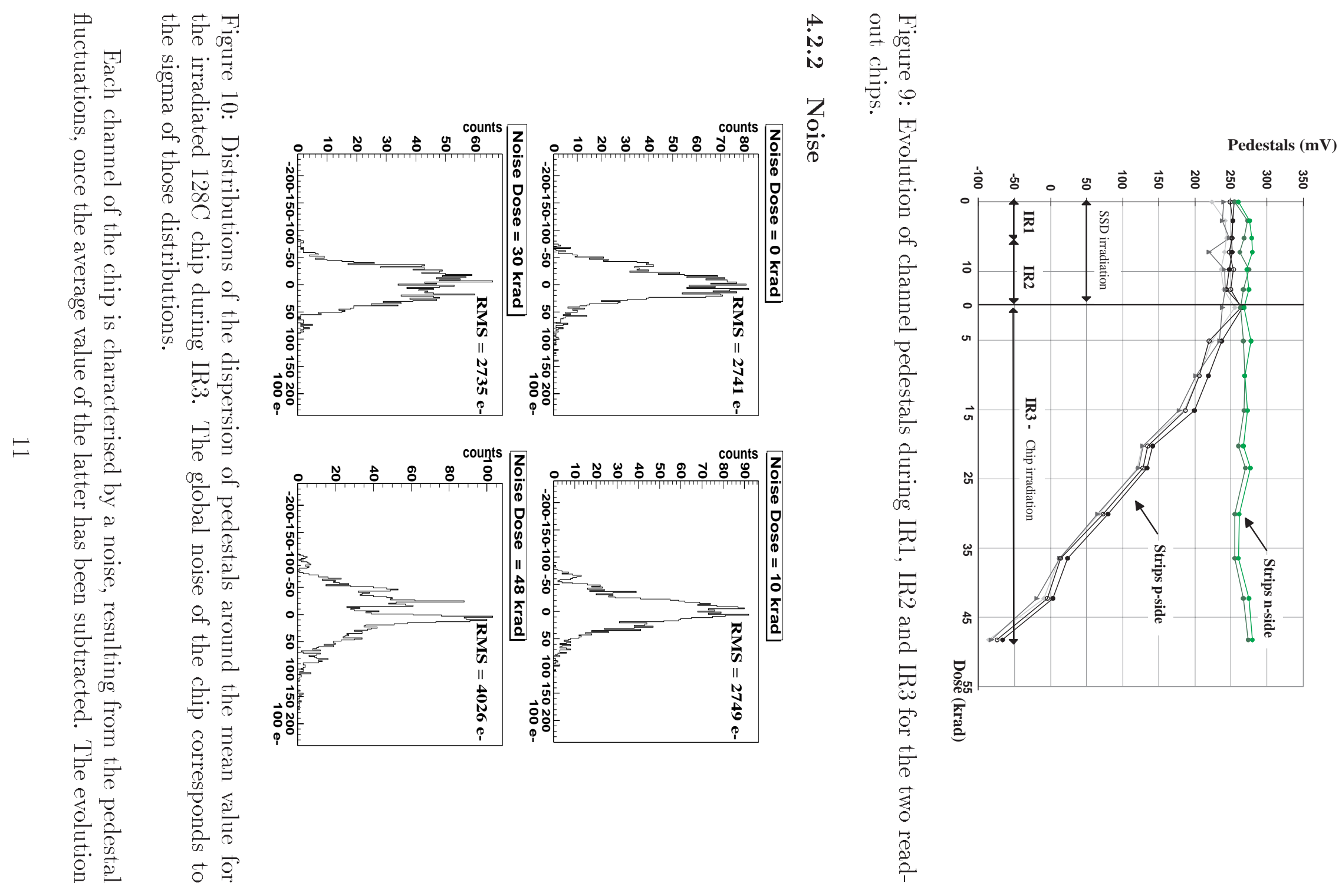
of the noise distribution, calculated for the 128 channels of the irradiated chip, is shown in Fig. 10. The data correspond to distributions measured at 4 different steps of IR3. A significant increase (47\%) of the distribution width occurs only after about $30 \mathrm{krad}$.

\subsubsection{Gain}

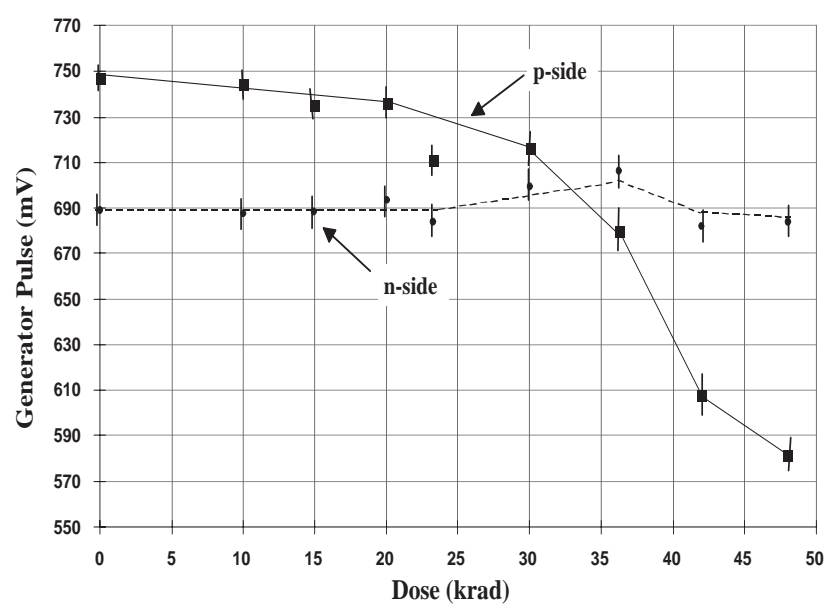

Figure 11: Evolution of the amplitude pulses from the internal generator for the $128 \mathrm{C}$ chip during IR3. The squares (rounds) corresponds respectively to the irradiated (non-irradiated) chip p-side (n-side). The errors bars correspond to the amplitude dispersion for the various fired channels.

A possible change in the gain of the chip during IR3 was examined. The analysis was performed by firing pulses, yielded by the internal generator ([3]) of the chips, in every twentieth channel. The results of the combination of the internal generator and the amplification chain are presented in Fig. 11, where the average amplitude of the pulses in the different channels of the two chips (both sides of the SSD) is plotted versus the absorbed dose over IR3. As it may be anticipated, the amplitudes remains constant for the no-irradiated chip (n-side of the SSD-structure). A decrease of the amplitude of $23 \%$ is measured for the exposed chip up to $50 \mathrm{krad}$. Here again the significant decrease occurs after about $30 \mathrm{krad}$. Taking into account the stability of noise up to $30 \mathrm{krad}$ (Fig. 10) and the small decrease of the pulses amplitudes $(\simeq 6 \%$ up to $30 \mathrm{krad}$ ), one should expect a maximum decrease of the cluster $\mathrm{S} / \mathrm{N}$, due to chip irradiation, of about $6 \%$ after $30 \mathrm{krad}$.

Like for the pedestals, a re-adjustment of the control parameters of the chip allows a restoration of the amplitudes. 


\subsubsection{Chip power supplies}

It is recognized [18] that in experiments at ultra-relativistic energies, particles may deposite a huge amount of energy (> 5000 MiP's) which may create local short-circuit, inducing a sudden high current which may damage the electronics chip. This phenomenon, so-called latchup, has to be examined carefully, even at the level of the SSD in the ITS of the ALICE Experiment, although it was not expected initially to be particularly troublesome there. The present instrumental setup is not appropriate to study this effect, and tests specifically devoted to it are currently in progress in another context. However, the bias current $i_{B+}$ and $i_{B-}$ have been recorded during IR3. If ever a latch-up should occur, it should generate a brutal high current intensity. The bias currents are plotted in Fig. 12 as a function of the absorbed dose during IR3. They exhibit a steady regime with 5 spikes. However, these brutal increases may be reliably related to DAQ failures and not to latchup. No more conclusion may be drawn about this problem at this point.

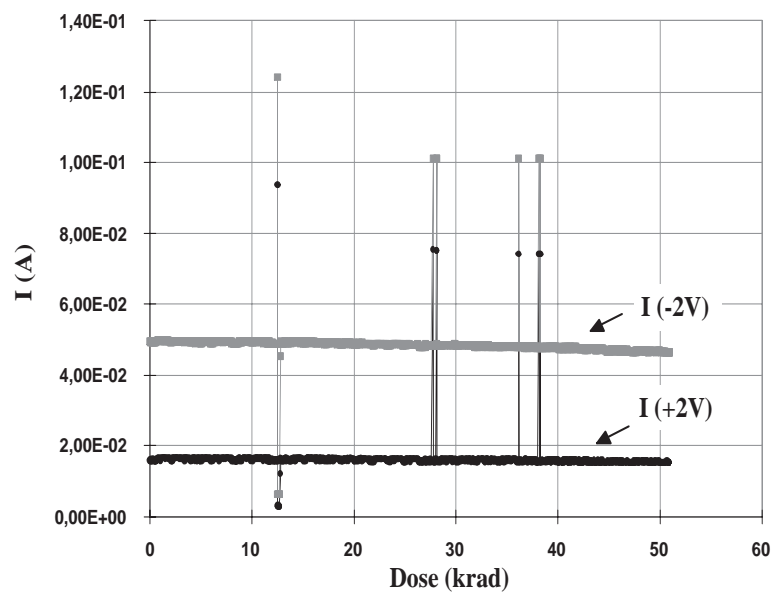

Figure 12: Bias currents $i_{B+}$ and $i_{B-}$ of the p-side $128 \mathrm{C}$ chip as a function of the absorbed dose for IR3.

\section{Conclusion}

In this work, we have studied the behaviour of a test-structure of a double-sided SSD bonded to two read-out ALICE 128C chips, both detector and chips at the ALICE-experiment specifications, submitted to the irradiation of $20 \mathrm{MeV}$ protons. The tests have been conducted in such a way that the SSD absorbed a total dose of $15 \mathrm{krad}$ (split in two exposures of different parts of the SSD, i.e. 5 and $10 \mathrm{krad}$ ), and the chip was exposed to a dose equivalent, for the SSD, to $50 \mathrm{krad}$. These figures exceed those expected in the whole ALICE experiment. Although an increase of the leakage current in the SSD is measured (64\%), comparable to those previously 
measured by other authors, it does not preclude a satisfactory operation of the detector since the main features, like signal to noise ratio and charge matching, remain essentially unaffected.

The characteristics of the chip appears to remain constant up to $30 \mathrm{krad}$. The degradation of those characteristics after $30 \mathrm{krad}$ induces however minor consequences on the global performances of the chip. Furthermore, the nominal values may be restored by control parameter adjustment.

In view of these results, this SSD + chip module may be considered as correctly operating and 320 units will be soon assemble to constitute an additional layer of detection around the Silicon Vertex Tracker of the STAR detector at RHIC.

\section{Acknowledgements}

We are indebted to Mrs S. Delacroix, of the "Centre de Protonthérapie d'Orsay (CPO)", for her participation to the ALANINE calibration.

\section{References}

[1] ALICE Technical Proposal, CERN/LHCC 95-71, LHCC/P3, December 1993

[2] M. Ayachi et al., Proc. of the Fourth Workshop on Electronics for LHC Experiments, Rome (1998)

[3] J.R. Lutz et al, ., Proc. of the Fourth Workshop on Electronics for LHC Experiments, Rome (1998)

[4] J.F. Ziegler et al, Monte Carlo program TRIM (The transport of Ions in Matter)

[5] J. W. Hansen et al., Radiat. Prot. Dosim. 27(1989)85 and references therein.

[6] F.F. Kuntz et al, Rad. Phys. Chem., to be published

[7] N. Bingefors, M. Burns, SIROCCO IV : front end readout processor for DELPHI microvertex. CERN/PPE 88-088

[8] D. Meier, Ph. D. Thesis, University of Heidelberg (1999) to be published

[9] C. Suire et al., ALICE Internal Note (1999)

[10] L. Hébrard et al., Proc of the Third Workshop on Electronics for LHC Experiments", Imperial College, London (1997)

[11] L. Hébrard et al., Proc. of the Fourth Workshop on Electronics for LHC Experiments, Rome (1998)

[12] John A.J. Matthews et al, ATLAS Internal Note, INDET-NO-118 (1995) 
[13] P.P. Allport et al., ATLAS Note, INDET-NO-192 (1997)

[14] D. Morgan et al., ATLAS Note, INDET-NO-199 (1998)

[15] John A.J. Matthews et al, CDF/DOC/SEC_VTX/PUBLIC/3937

[16] S. Di Liberto et al., ALICE Note, INT-NO-43 (1998)

[17] L. Arnold et al,ALICE/SIL 98-05, Internal Note-SIL (1998)

[18] F. Anghinolfi et al., RD49 status report, CERN-LHCC 97-63 (1997) 